(6) OPEN ACCESS

Correspondence to

Dr Simon Nadel, Department of Paediatric Intensive Care,

St Marys Hospital, Praed Street, London W21NY, UK;

s.nadel@imperial.ac.uk

Accepted 3 May 2012

Published Online First

15 September 2012

\title{
Prospects for eradication of meningococcal disease
}

\author{
Simon Nadel
}

\section{ABSTRACT}

Meningococcal meningitis and septicaemia remain a serious global health threat. This review focuses on the epidemiology of meningococcal disease following the recent implementation of effective vaccines and the potential utility of a vaccine against serogroup $B$ meningococcus.

\section{INTRODUCTION}

Meningococcal disease remains a serious global health threat associated with high mortality and morbidity, despite advances in antibiotic therapy, organ support techniques and modern vaccination strategies.

Young children and adolescents are the groups which suffer the greatest disease burden. Children are particularly vulnerable to meningococcal disease because of relative immaturity of their immune system, in particular their impaired immunity to the polysaccharide capsule of the meningococcus. ${ }^{1}$ It is estimated that over $75 \%$ of all cases of meningitis and septicaemia due to the meningococcus occur in children under 5 years of age, and these are some of the most common lifethreatening infections in children worldwide. Adolescents have the highest prevalence of nasopharyngeal carriage, and disease is also common in this age group. ${ }^{2}$

WHO estimates that about 170000 deaths occur annually from meningococcal and other bacterial meningitis and meningococcal septicaemia worldwide; the case fatality rate can be as high as $50 \%$, even with treatment. ${ }^{3}{ }^{4}$ In addition, the estimated median risk of at least one major or minor sequela from these infections after hospital discharge is $20 \%$ (range 12.3-35.3\%). The risk of adverse outcome varies with age group, geographical location and the infecting organism. In middle-income and low-income countries, meningococcal disease remains the fourth leading cause of disability. ${ }^{5}$

\section{BACTERIAL MENINGITIS}

Worldwide, the epidemiology of bacterial meningitis has changed dramatically in the last two decades following the introduction of new, highly effective conjugate protein/polysaccharide vaccines.

Prior to introduction of the conjugate vaccine against Haemophilus influenzae type $b$ (Hib), this was the most common cause of bacterial meningitis worldwide. ${ }^{6}$ More recently, introduction of similar effective vaccines against Streptococcus pneumoniae and serogroup C Neisseria meningitidis have also resulted in significant reductions in disease burden due to these organisms.

Despite these successes, international efforts to reduce the incidence of bacterial meningitis have taken on added impetus since recent epidemiological data revealed that meningococcus, Hib and pneumococcus are directly responsible for as many child deaths as HIV/AIDS, malaria and tuberculosis together. ${ }^{4}$

\section{EPIDEMIOLOGY OF MENINGOCOCCAL DISEASE}

$N$ meningitidis is an obligate human commensal living in the upper respiratory tract. The estimated nasopharyngeal carriage rate ranges from $0.6 \%$ to $34 \%$ and is higher in adolescents and individuals living in overcrowded and confined spaces. ${ }^{2} 7$

There are at least 13 known serogroups of $N$ meningitidis; however, more than $90 \%$ of disease is caused by serogroups A, B, C, W-135, X and Y. The distribution of serogroups varies with age group and geographical location. ${ }^{2}$

An estimated 500000 cases of meningococcal disease occur annually worldwide with a case fatality rate of at least $10 \% .^{8}$ Most cases occur during the winter months and early spring.

The incidence of meningococcal disease worldwide is difficult to ascertain accurately because of wide variation in surveillance in different regions of the world, together with under-reporting from many developing nations.

The incidence has decreased to $<1-3$ cases per 100000 population per year in the developed world. ${ }^{9}$

During pandemic meningococcal disease in sub-Saharan Africa, attack rates exceed 100-800 cases per 100000 population per year, with the highest attack rates reaching as high as 1 in $100 .^{9}$

The incidence of disease due to different serogroups is constantly changing, not only due to selection pressure caused by introduction of effective vaccines, but also due to stochastic variations in epidemiology.

Serogroup A meningococcus used to be very common in the UK before the first and second world wars; however, for reasons that are not clear, since the 1970s it has virtually disappeared as a cause of disease from Western Europe. Serogroup A meningococcus, however, remains the most common cause of meningococcal disease worldwide, and causes huge epidemics in the meningitis belt in sub-Saharan Africa, with an incidence as high as 1 case per 100 population, and case fatality rate reaching $75 \%$ in children and adolescents. ${ }^{4} 1011$ Serogroup A meningococcus is much less common in the developed world, although it is found in parts of China and Russia.

Serogroup B meningococcus (MenB) causes endemic disease in much of the developed world, including North America and Canada, Western Europe, Australasia and South America. Since the successful introduction of vaccines effective against serogroup $\mathrm{C}$ meningococcus (MenC) in 
much of the developed world, MenB now contributes approximately $80 \%$ of total disease burden in these areas, $50 \%$ of which occurs in children under 2 years of age. ${ }^{4} 1012$

Serogroup C meningococcus is also common in the developed world and is occasionally known to cause epidemics and outbreaks. ${ }^{4}{ }^{12-14}$ The incidence of MenC disease has decreased in those parts of the world, such as Western Europe and Canada, following the widespread introduction of effective conjugate vaccines against MenC.

Serogroup $Y$ meningococcus is becoming an increasingly important cause of meningococcal disease in the USA and is increasingly seen in the UK, even though numbers of cases are relatively small.

Serogroup $X$ meningococcus is becoming increasingly common in parts of Africa. ${ }^{4}$

There were over 1000 cases of meningococcal disease reported in England and Wales in the year 2010-2011. ${ }^{15}$ The majority were due to MenB and over $80 \%$ of cases were in children under 5 years.

There were approximately 300 children admitted to paediatric intensive care units in the year 2009-2010, with approximately 60 deaths (Dr Phil McShane, PICANet, personal communication).

It is estimated that up to $25 \%$ of survivors of meningococcal disease will have significant morbidity, including amputation, skin loss, orthopaedic abnormalities, nerve deafness, other neurological abnormalities including hemiplegia, neurodevelopmental delay and epilepsy. ${ }^{16}$ In addition, a large number of affected children and their families will suffer profound neuropsychological consequences of this devastating disease, including post-traumatic stress disorder, depression, psychosis, reduced educational performance and major anxiety. ${ }^{17}$

An effective vaccine against the most prevalent cause of meningococcal disease, MenB, is likely to significantly reduce this disease burden and would have a major impact on the morbidity and mortality caused by MenB.

\section{VACCINES FOR MENINGOCOCCAL DISEASE}

There are two main types of vaccines used for protection against meningococcal infection: pure polysaccharide vaccines and protein/polysaccharide conjugate vaccines. These are based on the capsular polysaccharide of the bacteria, which is a major virulence factor and is responsible for prevention of host-mediated bacterial killing.

The immunogenic response to pure polysaccharide vaccines is diminished in infants and young children under the age of 2 years because of reduced immunogenicity of T-cell-independent antigens such as polysaccharides. This is an important limitation given that the highest disease burden is in young children and infants. In addition, these vaccines do not prevent acquisition of nasopharyngeal carriage or confer herd immunity, ${ }^{18}$ and vaccine responses with repeated administration over time (eg, with immunisations of at-risk populations during repeated epidemics) is uncertain. ${ }^{19} 20$

The pure polysaccharide quadrivalent vaccine against serogroups A, C, Y and W-135 is also poorly immunogenic in children under 2 years of age, and gives only temporary immunity, lasting for approximately 3-5 years in older people; it does not have any effect on nasopharyngeal carriage of the meningococcus and it has now been superceded by a conjugate quadrivalent vaccine. ${ }^{10} 21 \quad 22$ Effective quadrivalent conjugate polysaccharide vaccines have become available against serogroups A, C, Y and W-135. These multivalent MenACWY vaccines have proven to be effective in infants and older subjects.
In the USA, the Advisory Committee on Immunisation Practices recommended routine vaccination of young adolescents beginning at age 11 years. Routine vaccination with this vaccine is also recommended for college freshmen living in dormitories and for other populations at increased risk (ie, military recruits, travellers to areas in which meningococcal disease is hyperendemic or epidemic, microbiologists who are routinely exposed to isolates of $N$ meningitidis, patients with anatomic or functional asplenia, and patients with terminal complement deficiency). ${ }^{23}$ Because of the relatively low incidence of serogroups A, Y and W-135 meningococcal disease in the UK, especially in children, routine quadrivalent ACW135Y conjugate vaccination is unlikely to be cost effective in the UK.

As serogroup A meningococcal disease is a major public health concern in the meningitis belt of sub-Saharan Africa, the WHO initiated the Meningitis Vaccine Project, which has developed a low-cost conjugate vaccine against serogroup A (MenAfriVac). ${ }^{4} 24$ This vaccine has been successfully tested in phase I, II and III clinical trials and has been launched in mass vaccination campaigns as a single dose to a target population of 250 million people aged 1-29 years across 25 countries in the African meningitis belt. More than 1 million people have already received the vaccine in the region.

Serogroup C meningococcal conjugate vaccine has been introduced successfully into several countries in Europe as a part of the routine immunisation schedule in infants. ${ }^{11} 21$ This vaccine is not only strongly immunogenic, giving relatively long-lasting immune response and immunological memory, but it also confers herd immunity by decreasing nasopharyngeal carriage.

Since the introduction of MenC conjugate vaccine into the UK in 1999, the incidence of MenC disease has decreased by $94 \%$ in immunised populations and $67 \%$ in unimmunised populations. In addition, a significant decrease in nasopharyngeal carriage has been reported, with no increase in the carriage of other serogroups. ${ }^{25}$

Following MenC conjugate vaccination in infancy there is waning of post-vaccination functional antibody titres by the 12th month of life, which has led to the introduction of the booster dose in the second year of life in the UK schedule. ${ }^{26} 27$

The UK Joint Committee on Vaccination and Immunisation (JCVI) has noted the above studies showing that levels of antibody considered to be protective, wane rapidly in infants and children aged under 6 years who were vaccinated with the MenC vaccine, such that only approximately $12 \%$ are considered to have protective levels 4 years after vaccination. ${ }^{26}$ However, if children are immunised when they are over 6 years of age, then around $50 \%$ still have protective levels of antibody in early adolescence. ${ }^{28}$ In contrast, antibody levels considered to be protective in individuals vaccinated from the age of 10 years or older are markedly higher, and protection therefore persists until at least early adulthood and possibly longer.

The JCVI concluded that herd immunity could be maintained by introducing a booster dose in older children to increase protective antibody levels, and advised that a booster dose of MenC vaccine should be provided during adolescence. It also recommended that one of the doses is removed in infancy. ${ }^{29}$

A conjugate ACWY vaccine could be an option in older children if the incidence of other vaccine-preventable disease increased. These quadrivalent vaccines could also play a significant role in the national schedules of the developed world as an adolescent booster to provide wider serogroup coverage. 


\section{The problem of serogroup B $\mathbf{N}$ Meningitidis}

Serogroup B meningococcus remains a common cause of meningococcal septicaemia and meningitis, and accounts for more than $50 \%$ of cases in the USA and as many as $90 \%$ of cases in Europe since the introduction of the conjugated MenC vaccine. $^{3031}$

Serogroup B meningococcus has a poorly immunogenic capsule, which has hindered progress on developing a polysaccharide vaccine effective against it. ${ }^{32}$ The polysaccharide capsule of MenB is composed of polysialic acid $\left(\alpha^{2-8}\right.$ $\mathrm{N}$-acetylneuraminic acid) which is present in many human glycoproteins; in particular, it is similar to carbohydrates found in fetal brain tissue. Therefore there is some immune tolerance to this polysaccharide, and there are concerns regarding the effect of modifying the sugar structure in a vaccine to make it immunogenic in case of induction of auto-immunity. This mimicry therefore does not allow the use of protein/polysaccharide conjugate vaccine.

Subsequently, vaccine development for MenB has required a different approach from that used for the preparation of conjugate vaccines for serogroups A, C, Y and W-135.

Because of the difficulty in producing an effective capsular vaccine against MenB, vaccines have now been developed targeting non-capsular structures, such as outer membrane porins, vesicles and lipopolysaccharide (LPS). ${ }^{33-35}$

LPS is a universal component of the outer membrane of Gram-negative bacteria, and contributes to resistance of $N$ meningitidis to complement. ${ }^{36}$ In addition, $N$ meningitidis produces a surface protein that binds human complement factor $\mathrm{H}$ (factor $\mathrm{H}$ binding protein, $\mathrm{fHbp}$ ). Factor $\mathrm{H}$ is important in regulating the alternative complement pathway and therefore its binding to the surface of meningococci is thought to inhibit complement-mediated bacterial lysis. This is therefore a protective factor for the bacteria, promoting bacterial survival.

Recently, it has been demonstrated that another surface protein, called Neisserial surface protein A (NspA), is also able to bind human complement factor $\mathrm{H}(\mathrm{fH}) .{ }^{37}$ Other factors which mimic or bind host molecules also function to prevent complement-mediated bacterial lysis and phagocytosis. For instance, $N$ meningitidis sheds outer membrane blebs containing proteins and LPS. These outer membrane vesicles (OMVs) are able to initiate complement activation and might thereby redirect complement activation away from whole cell meningococci in the circulation, hindering the bactericidal effects of complement. $^{36}$

Because of the difficulties in producing an effective vaccine against MenB, various strategies have been proposed, some of them being introduced into clinical practice.

The development of MenB vaccines has focused on subcapsular antigens, either as OMVs or as individual antigens.

OMV can be separated from meningococcal bacteria or isolated as membrane blebs, which are released during bacterial growth. The OMVs have been shown to contain four or five major outer membrane proteins (OMPs) and various other periplasmic and cytoplasmic proteins. The role of these proteins in safety or immunogenicity is unknown. ${ }^{32}$

Several candidate OMV vaccines have been developed and tested in large-scale efficacy studies in Norway, Cuba, Brazil, Chile and New Zealand, with variable results. A Norwegian study conducted in 1988 on 171800 students in secondary schools who volunteered to take part in a double-blind, placebo-controlled, efficacy trial demonstrated a rate of protection of $57.2 \%{ }^{38}$ These findings suggested that, although the vaccine conferred protection against MenB disease, the effect was insufficient to justify a public vaccination programme.

Good results were reported in a Cuban study of OMPs from MenB. ${ }^{39}$ Efficacy was tested on 106000 10-14-year-old students and was estimated at $83 \%$. These results and the fact that no severe or long-lasting reactions to the vaccine were observed prompted the Cuban Ministry of Public Health to vaccinate all children aged between 3 months and 6 years in the most severely affected provinces. The efficacy of vaccination varied from $83 \%$ to $94 \%$. After 3 years of a widespread programme, no severe reactions occurred, and one of the most severe epidemics was practically eradicated.

Unfortunately, these results were only partially confirmed in other epidemics occurring in other nations such as Brazil and Chile. Since these vaccines are based on a single meningococcal isolate, they can provide only partial protection against virulent heterologous meningococci, and the degree of protection appears to be dependent on age. ${ }^{40-43}$

These findings are particularly relevant in countries where MenB disease is of a multiclonal nature, such as The Netherlands and the UK.

Furthermore, it is clear that the OMV vaccines can be useful in curtailing localised epidemics through the administration of a specific 'tailor-made' vaccine, as the New Zealand experience subsequently demonstrated. ${ }^{44}$ Since 1991, an epidemic of MenB infection has afflicted New Zealand, causing more than 4700 cases and over 200 deaths. ${ }^{45}$ The overall incidence peaked in 2001 at 17.4 cases per 100000 people. In 2002, Maori and Pacific Island children under 1 year of age displayed incidence rates of 286 and 368 per 100000 , respectively. On the advice of WHO, an international advisory group was set up in 1993 and several options were considered; in 2000, the New Zealand health authorities accepted a proposal by the Norwegian Institute of Public Health and Chiron Vaccines (Siena, Italy) to develop a vaccine and to implement clinical trials. This approach involved preparing a protein-based OMV vaccine from a wild-type strain typical of the one responsible for the epidemic. ${ }^{46}$ A new strain-specific MenB vaccine, referred to as 'MeNZB', was developed and its effectiveness was assessed in a prospective observational study following a nationwide vaccination programme in New Zealand. The vaccination programme began in July 2004, and the study used data from January 2001 to June 2006. Fully vaccinated children under 5 years of age were up to six times less likely to contract epidemic strain meningococcal disease in the 24 months after vaccination, corresponding to an estimated vaccine effectiveness of $80 \% .{ }^{47}$ The OMV vaccines are useful for control of epidemics because they are directed against specific surface proteins, which are antigenically variable; therefore, the vaccines can be tailored to a predominant strain during an epidemic. However, these vaccines do not confer cross-protective immunity against other strains of MenB and therefore are geographically limited in their utility.

\section{REVERSE VACCINOLOGY IN THE SEARCH FOR A UNIVERSAL VACCINE AGAINST SEROGROUP B MENINGOCOCCUS}

To develop a universal vaccine against $\mathrm{MenB}$, researchers at Novartis Vaccines used the results of the genomic sequence of a serogroup B strain ( $N$ meningitidis serogroup B strain MC58). ${ }^{48}$ Thus, MenB became the prototype for the use of genomics for vaccine development, a process called 'reverse vaccinology'.

This approach predicted around 600 novel antigens, the candidate sequences of which were expressed in Escherichia coli and used to immunise mice. 
Analysis of sera revealed more than 90 previously unknown bacterial surface-located proteins, 29 of which were able to induce bactericidal antibodies. ${ }^{49}$

Subsequently, several antigens were selected and included in a new prototype vaccine. Neisserial heparin binding antigen, fHbp and NadA (an antigen which promotes adhesion to and invasion of epithelial cells) were included..$^{50}$

In 2008, Novartis Vaccines initiated clinical trials of this new vaccine, named $4 \mathrm{CMenB}$ (four-component meningococcal $\mathrm{B}$ vaccine), with the OMV of the New Zealand vaccine strain, in adolescents and infants. The addition of the OMV increased strain coverage because the immunogenic PorA antigen is present within the outer-membrane vesicle.

In a phase II trial, 147 infants were vaccinated with three doses at 2, 4 and 6 months of age. Safety and immunogenicity were good, with adequate serum bactericidal titres against reference strains in $87 \%, 85 \%$ and $95 \%$ of subjects after the third dose; these values increased to $100 \%, 96 \%$ and $93 \%$, respectively, after a booster dose administered at 12 months, this finding indicating induction of immunological memory. ${ }^{51}$ The vaccine displayed good safety and tolerability. Subsequent studies in the 2, 3 and 4 month age groups with a booster at 12 months has shown similar effectiveness. ${ }^{52}$

This vaccine is now being studied in several phase III studies. Immunogenicity studies suggest that this vaccine given in infants and in adolescents provides broad coverage against the strains represented in the vaccine components, although there remains uncertainty about the actual coverage that would be achieved once the vaccine is introduced into the routine schedule. 5253

The adolescent study in 1631 subjects showed that protective antibody titres against three MenB strains developed in over $90 \%$ of participants after one dose, increasing to $99-100 \%$ when two doses were administered at intervals of 1 month, 2 months or 6 months. A third dose of $4 \mathrm{CMenB}$ provided no additional immunological benefit. Local and systemic reaction rates were similar after each $4 \mathrm{CMenB}$ injection and did not increase with subsequent doses, but remained higher than placebo. No vaccine-related serious adverse events were reported and no significant safety signals were identified. ${ }^{53}$

Another study in 1800 healthy infants showed that when administered together with routine vaccines, a primary immunisation course of $4 \mathrm{CMenB}$ is immunogenic against three reference strains expressing one of three vaccine antigens. Furthermore, 4CMenB was immunogenic when administered in a schedule of 2, 3 and 4 months, an important finding given the high rates of MenB disease in the first 6 months of life. These results suggest that there can be some flexibility in the incorporation of $4 \mathrm{CMenB}$ into the various immunisation schedules used in different countries. ${ }^{52}$

Current estimates suggest around $78 \%$ of circulating strains in the UK would be covered by this vaccine. ${ }^{54}$ However, this estimate has to be treated with caution as the assay on which it is based is a surrogate assay (meningococcal antigen typing system (MATS)) of the serum bactericidal antibody (SBA) assay, and the correlation of MATS with the SBA has not definitively been established. ${ }^{55}$ In addition, the MATS assay is based on a limited panel of strains which may not be directly applicable to the diverse strains causing disease in the UK. Ultimately, the exact coverage of this vaccine will not be known until it is widely used in diverse populations.

Additionally, although coadministration of $4 \mathrm{CMenB}$ with other routine infant vaccines has induced an increased incidence of systemic reactions in infants, possibly due to the presence of LPS in the OMV component of the vaccine, it appears that coadministration with paracetamol produces an acceptable tolerability profile, with no obvious loss in immunogenicity in $4 \mathrm{CMenB}$ or other routine coadministered infant vaccines. ${ }^{56}$ These data are not yet peer reviewed and have to be interpreted with caution. The use of paracetamol concomitantly with vaccination is controversial since a recently published study suggests that coadministration might affect immunogenicity of routine vaccines. ${ }^{57}$ In this study, antibody concentrations after primary vaccination for infants receiving routine vaccination were significantly lower in the prophylactic paracetamol group than in the no-prophylactic paracetamol control group.

On 23 December 2010, Novartis Vaccines and Diagnostics applied to the European Medicines Agency for authorisation to market this multicomponent vaccine for MenB. Decisions regarding licensure and possible integration into the UK vaccine schedule could follow later this year. One factor that is always taken into account when assessing whether a new vaccine is introduced into the schedule is cost effectiveness. The $4 \mathrm{CMenB}$ vaccine is expensive and calculations regarding cost-effectiveness have produced conflicting results. Novartis have yet to announce the exact cost of this vaccine for the costeffectiveness analysis.

\section{OTHER STRATEGIES}

Other vaccines against MenB containing recombinant fHbp, along with other components of the bacterial outer membrane or cell wall, are undergoing clinical trials. ${ }^{58}$

At the same time as Novartis were developing their MenB vaccine, Pfizer (formerly Wyeth) independently identified fHbp as an important antigen and developed a vaccine containing two fHbp variants. ${ }^{59}$

A vaccine containing antigenic components from subfamilies $\mathrm{A}$ and $\mathrm{B}$ of meningococcal $\mathrm{fHbp}$ has been evaluated in phase II studies which have concluded that this bivalent vaccine elicited bactericidal activity against subfamily A and B strains in a high proportion of adults and adolescents, and that no significant safety concerns were identified. ${ }^{60}$ Animal studies suggested that about $87 \%$ of MenB strains will be covered by such a vaccine. ${ }^{61}$ Further evaluation of this vaccine is currently underway.

While fHbp is present in all meningococci, its degree of expression differs among isolates. ${ }^{59}{ }^{62}$ In most meningococcal strains, its epitope surface exposure is less than that of other vaccine antigens. ${ }^{63}$ Therefore its use as a vaccine antigen may be limited. However, fHbp expression is a prerequisite to the survival of meningococci, especially of high-expressing strains in ex vivo human blood and serum, so it is likely to be an ideal vaccine candidate. ${ }^{59} 64$

Vaccines against all serogroups of $N$ meningitidis are likely to be licensed in the near future. However, some important questions remain: their effectiveness on the different geographical distribution of pathogenic strains, their impact on carriers and the timing of the appearance of escape mutants once herd immunity has been achieved.

One of the most important issues to be determined is the impact on asymptomatic nasopharyngeal meningococcal carriage. Asymptomatic carriage of $N$ meningitidis is common (detected in $5-35 \%$ of individuals). ${ }^{65}$ The prevalence of carriage is low in infancy, increases during childhood, and reaches its peak in adolescents and young adults; thereafter, it declines in adults and older people. ${ }^{66}$ 
Studies regarding carriage in vaccinees who have received $4 \mathrm{CMenB}$ vaccine are being carried out to establish whether this vaccine has any effect on nasopharyngeal carriage, a prerequisite for the establishment of herd immunity (R Borrow, personal communication). However, the only way to truly assess the effects of these vaccines on herd immunity is to carry out increased surveillance following vaccine introduction. It is important to consider the possibility that the MenB vaccines would have no impact on nasopharyngeal carriage, in which case there would be no clear strategy to induce herd immunity. If there is an impact on nasopharngeal carriage, then strategies that target carriers such as immunisation of adolescents or adults could be appropriate.

Another potential benefit of outer-membrane protein vaccines is their potential to induce cross protection against other non-MenB strains. A recent study showed that the FHbp gene was present in all MenC isolates from patients with MenC disease in the USA, suggesting an FHbp-based vaccine may also be effective in protecting against a diverse range of meningococcal serogroups causing disease. ${ }^{67}$ This raises the possibility of a universal meningococcal vaccine, potentially leading to eradication of meningococcal disease.

\section{CONCLUSION}

Development and implementation of effective MenB vaccines are likely to produce significant health benefits, both in prevention of critical illness, and long-term disabling sequelae after meningitis and septicaemia.

Everything that can be done to reduce the consequences of meningococcal disease, including improvements in recognition and management, improvements in diagnostics, and most importantly, prevention of disease, is essential to improving the life chances of children and their families. It is important that any delays to implementation of new MenB vaccines are minimised to ensure children are protected from this devastating disease as soon as effective vaccines become available.

Acknowledgement SN is grateful to the Comprehensive Biomedical Research Centre at Imperial College Healthcare NHS Trust for support.

Contributors SN conceived of and wrote this review.

\section{Competing interests Obtained.}

Provenance and peer review Not commissioned; externally peer reviewed.

\section{REFERENCES}

1. Davies EG. The immunology of neonates and children and its relation to susceptibility to infection. In: Nadel S, ed. Infectious Diseases in the Pediatric Intensive Care Unit. Berlin: Springer, 2008:1-58.

2. Stephens DS, Greenwood B, Brandtzaeg P. Epidemic meningitis, meningococcaemia, and Neisseria meningitidis. Lancet 2007;369:2196-210.

3. World Health Organization. New and under-utilized vaccines implementation (NUVI): bacterial meningitis (online). http://www.who.int/nuvi/meningitis/en/index. $\mathrm{html}$ (accessed 13 Apr 2011).

4. World Health Organization. Meningococcal meningitis (online). http://www.who. int/mediacentre/factsheets/fs141/en/index.html (accessed 18 Feb 2011).

5. Edmond K, Clark A, Korczak VS, et al. Global and regional risk of disabling sequelae from bacterial meningitis: a systematic review and meta-analysis. Lancet Infect Dis 2010;10:317-28

6. Martin M, Casellas JM, Madhi SA, et al. Impact of Haemophilus influenzae type b conjugate vaccine in South Africa and Argentina. Pediatr Infect Dis J 2004;23:842-7.

7. Rosenstein NE, Perkins BA, Stephens DS, et al. Meningococcal disease. N Eng/ J Med 2001;344:1378-88.

8. Centers for Diseases Control and Prevention. Meningococcal disease: technical and clinical information (online). http://www.cdc.gov/meningitis/clinical-info.html (accessed 17 Aug 2011).

9. Harrison LH, Trotter CL, Ramsay ME. Global epidemiology of meningococcal disease. Vaccine 2009;24:B51-63.
10. Centers for Diseases Control and Prevention. Active Bacterial Core surveillance (ABCs) report: emerging infections program network. Neisseria meningitidis, 2006 (online). http://www.cdc.gov/abcs/reports-findings/survreports/mening06.pdf (accessed 17 Aug 2011).

11. Stephens DS. Conquering the meningococcus. FEMS Microbiol Rev 2007;31:3-14.

12. The European Union Invasive Bacterial Infections Surveillance Network. Invasive meningococcal disease (online). http://www.ecdc.europa.eu/en/publications/ Publications/1011_SUR_Annual_Epidemiological_Report_on_Communicable_ Diseases in Europe.pdf\#page $=149$ (accessed 21 Feb $201 \overline{1}$ )

13. EU-IBIS Network. Invasive Neisseria Meningitidis in Europe 2006. London: Health Protection Agency, 2006. http://www.hpa-bioinformatics.org.uk/euibis/documents/ 2006 meningo.pdf (accessed 17 Aug 2011).

14. Harrison LH. Epidemiological profile of meningococcal disease in the United States. Clin Infect Dis 2010;50:S37-44.

15. Health Protection Agency. http://www.hpa.org.uk/hpr/infections/immunisation.htm (accessed 24 Feb 2012).

16. Wright C. Counting the cost of meningitis. Presented at the Meningitis Research Foundation Conference 2011. November 8th/9th 2011, London, UK: Royal Society of Medicine.

17. Garralda ME, Gledhill J, Nadel S, et al. Longer-term psychiatric adjustment of children and parents after meningococcal disease. Pediatr Crit Care Med 2009;10:675-80.

18. Hassan-King MK, Wall RA, Greenwood BM. Meningococcal carriage, meningococcal disease and vaccination. J Infect 1988;16:55-9.

19. Borrow R, Joseph Andrews N, et al. Reduced antibody response to revaccination and men with meningococcal serogroup A polysaccharide vaccine in adults. Vaccine 2001;19:1129-32.

20. Richmond $\mathbf{P}$, Kaczmarski E, Borrow R, et al. Meningococcal C polysaccharide vaccine induces immunologic hyporesponsiveness in adults that is overcome by meningococcal C conjugate vaccine. J Infect Dis 2000;181:761-4.

21. Girard MP, Preziosi MP, Aguado MT, et al. A review of vaccine research and development: meningococcal disease. Vaccine 2006;24:4692-700.

22. Centers for Diseases Control and Prevention. Prevention and control of meningococcal disease. Recommendations of the Advisory Committee on Immunization Practices (ACIP). MMWR Recomm Rep 2005;54:1-21.

23. Bilukha 00, Rosenstein N. Prevention and control of meningococcal disease. Recommendations of the advisory committee on immunization practices (ACIP). MMWR Recomm Rep 2005;54:1-21.

24. World Health Organization. Meningitis A conjugate vaccine. Wkly Epidemiol Rec 2011;86:42-3.

25. Ramsay ME, Andrews NJ, Trotter CL, et al. Herd immunity from meningococcal serogroup $C$ conjugate vaccination in England. BMJ 2003;326:365-6.

26. Borrow R, Goldblatt D, Andrews N, et al. Antibody persistence and immunological memory at age 4 years after meningococcal group $C$ conjugate vaccination in children in the United Kingdom. J Infect Dis 2002;186:1353-7.

27. Snape MD, Kelly DF, Green B, et al. Lack of serum bactericidal activity in preschool children two years after a single dose of serogroup $C$ meningococcal polysaccharide-protein conjugate vaccine. Pediatr Infect Dis J 2005;24:128-31.

28. Snape MD, Kelly DF, Lewis S, et al. Seroprotection against serogroup C meningococcal disease in adolescents in the United Kingdom: observational study. BMJ 2008;336:1487-91.

29. JCVI statement on the use of meningococcal $\mathbf{C}$ vaccines in the routine childhood immunisation programme. 29 January 2012.

30. Wenger JD, Hightower AW, Facklam RR, et al. Bacterial meningitis in the United States, 1986: report of a multistate surveillance study. The Bacterial Meningitis Study Group. J Infect Dis 1990;162:1316-23.

31. Gray SJ, Trotter CL, Ramsay ME, et al. Epidemiology of meningococcal disease in England and Wales 1993/94 to 2003/2004: contribution and experiences of the Meningococcal Reference Unit. J Med Microbiol 2006;55:887-96.

32. Panatto D, Amicizia D, Lai PL, et al. Neisseria meningitidis B vaccines. Expert Rev Vaccines 2011;10:1337-51.

33. Oliver KJ, Reddin KM, Bracegirdle $P$, et al. Neisseria lactamica protects against experimental meningococcal infection. Infect Immun 2002;70:3621-6.

34. Keiser PB, Biggs-Cicatelli S, Moran EE, et al. A phase 1 study of a meningococcal native outer membrane vesicle vaccine made from a group $B$ strain with deleted IpxL1 and synX, over-expressed factor $\mathrm{H}$ binding protein, two PorAs and stabilized OpcA expression. Vaccine 2011;29:1413-20.

35. Zollinger WD, Donets MA, Schmiel DH, et al. Design and evaluation in mice of a broadly protective meningococcal group B native outer membrane vesicle vaccine. Vaccine 2010;28:5057-67.

36. Schneider MC, Exely RM, Ram S, et al. Interactions between Neisseria meningitidis and the complement system. Trends Microbiol 2007;15:233-40.

37. Lewis LA, Ngampasutadol J, Wallace $\mathrm{R}$, et al. The meningococcal vaccine candidate neisserial surface protein $\mathrm{A}(\mathrm{NspA})$ binds to factor $\mathrm{H}$ and enhances meningococcal resistance to complement. PLoS Pathog 2010;6:e1001027.

38. Bjune G, Høiby EA, Grønnesby JK, et al. Effect of outer membrane vesicle vaccine against group B meningococcal disease in Norway. Lancet 1991;338:1093-6. 
39. Sierra GV, Campa HC, Varcacel NM, et al. Vaccine against group B Neisseria meningitidis: protection trial and mass vaccination results in Cuba. NIPH 1991:14:195-207.

40. Milagres LG, Gorla MC, Sacchi CT, et al. Specificity of bactericidal antibody response to serogroup B meningococcal strains in Brazilian children after immunization with an outer membrane vaccine. Infect Immun 1998;66:4755-61.

41. Milagres LG, Gorla MC, Rebelo MC, et al. Bactericidal antibody response to Neisseria meningitidis serogroup $B$ in patients with bacterial meningitis: effect of immunization with an outer membrane protein vaccine. FEMS Immunol Med Microbiol 2000;28:319-27.

42. Tappero JW, Lagos R, Ballesteros AM, et al. Immunogenicity of 2 serogroup B outer-membrane protein meningococcal vaccines: a randomized controlled trial in Chile. JAMA 1999;281:1520-7.

43. Wedege $\mathbf{E}$, Høiby EA, Rosenqvist $\mathrm{E}$, et al. Immune responses against major outer membrane antigens of Neisseria meningitidis in vaccinees and controls who contracted meningococcal disease during the Norwegian serogroup B protection trial. Infect Immun 1998;66:3223-31.

44. Oster P, Lennon D, O'Hallahan J, et al. MeNZB: a safe and highly immunogenic tailor-made vaccine against the New Zealand Neisseria meningitidis serogroup B disease epidemic strain. Vaccine 2005;23:2191-6.

45. Devoy AF, Dyet $\mathrm{KH}$, Martin DR. Stability of PorA during a meningococcal disease epidemic. J Clin Microbiol 2005;43:832-7.

46. Holst J, Feiring B, Fuglesang JE, et al. Serum bactericidal activity correlates with the vaccine efficacy of outer membrane vesicle vaccines against Neisseria meningitidis serogroup B disease. Vaccine 2003;21:734-7.

47. Galloway $\mathbf{Y}$, Stehr-Green P, McNicholas A, et al. Use of an observational cohort study to estimate the effectiveness of the New Zealand group B meningococcal vaccine in children aged under 5 years. Int $\mathrm{J}$ Epidemiol 2009;38:413-18.

48. Tettelin H, Saunders NJ, Heidelberg J, et al. Complete genome sequence of Neisseria meningitidis serogroup B strain MC58. Science 2000;287:1809-15.

49. Sette A, Rappuoli R. Reverse vaccinology: developing vaccines in the era of genomics. Immunity 2010;33:530-41.

50. Giuliani MM, Adu-Bobie J, Comanducci M, et al. A universal vaccine for serogroup B meningococcus. Proc Natl Acad Sci USA 2006;103:10834-9.

51. Findlow J, Borrow R, Snape MD, et al. Multicenter, open-label, randomized phase II controlled trial of an investigational recombinant Meningococcal serogroup B vaccine with and without outer membrane vesicles, administered in infancy. Clin Infect Dis 2010:51:1127-37.

52. Gossger N, Snape MD, Yu LM, et al. Immunogenicity and tolerability of recombinant serogroup $B$ meningococcal vaccine administered with or without routine infant vaccinations according to different immunization schedules: a randomized controlled trial. JAMA 2012;307:573-82.
53. Santolaya ME, O'Ryan ML, Valenzuela MT, et al. Immunogenicity and tolerability of a multicomponent meningococcal serogroup $B(4 \mathrm{CMenB})$ vaccine in healthy adolescents in Chile: a phase $2 \mathrm{~b} / 3$ randomised, observer-blind, placebo-controlled study. Lancet 2012;379:617-24.

54. Donnelly J, Medini D, Giuliani M, et al. Estimating the potential strain coverage in Europe of a multicomponent vaccine targeting serogroup B meningococci. Presented at Meningitis Research Foundation Conference 2011; 8-9 November 2011; London.

55. Donnelly J, Medini D, Boccadifuoco G, et al. Qualitative and quantitative assessment of meningococcal antigens to evaluate the potential strain coverage of protein-based vaccines. Proc Natl Acad Sci USA 2010;107:19490-5.

56. Peter Dull. Novartis vaccines. Presented at: Meningitis Research Foundation Conference 2011; 8-9 November 2011; London.

57. Prymula R, Siegrist CA, Chlibek R, et al. Effect of prophylactic paracetamol administration at time of vaccination on febrile reactions and antibody responses in children: two open-label, randomised controlled trials. Lancet 2009;374:1339-50.

58. Sandbu S, Feiring B, Oster $\mathrm{P}$, et al. Immunogenicity and safety of a combination of two serogroup B meningococcal outer membrane vesicle vaccines. Clin Vaccine Immunol 2007:14:1062-9.

59. Brehony C, Wilson DJ, Maiden MC. Variation of the factor H-binding protein of Neisseria meningitidis. Microbiology 2009;155:4155-69.

60. Richmond $\mathbf{P}$, Marshall H, Sheldon E, et al. Safety and immunogenicity of serogroup B Neisseria meningitidis (MnB) rLP2086 vaccine in adults and adolescent subjects: overview of 3 clinical trials. Presented at The 17th International Pathogenic Neisseria Conference; 11-16 September 2010; Banff, Canada.

61. Jiang HO, Hoiseth SK, Harris SL, et al. Broad vaccine coverage predicted for a bivalent recombinant factor $\mathrm{H}$ binding protein based vaccine to prevent serogroup $\mathrm{B}$ meningococcal disease. Vaccine 2010;28:6086-93.

62. Masignani V, Comanducci $\mathrm{M}$, Giuliani MM, et al. Vaccination against Neisseria meningitidis using three variants of the lipoprotein GNA1870. J Exp Med 2003:197:789-99.

63. Welsch JA, Rossi R, Comanducci M, et al. Protective activity of monoclonal antibodies to genome-derived neisserial antigen 1870, a Neisseria meningitidis candidate vaccine. J Immunol 2004;172:5606-15.

64. Seib KL, Serruto D, Oriente F, et al. Factor H-binding protein is important for meningococcal survival in human whole blood and serum and in the presence of the antimicrobial peptide LL-37. Infect Immun 2009;77:292-9.

65. Horton RE, Stuart J, Christensen $\mathrm{H}$, et al. Influence of age and carriage status on salivary IgA to Neisseria meningitidis. Epidemiol Infect 2005;133:883-9.

66. Christensen H, May M, Bowen L, et al. Meningococcal carriage by age: a systematic review and meta-analysis. Lancet Infect Dis 2010;10:853-61.

67. Harris SL, Zhu D, Murphy E, et al. Preclinical evidence for the potential of a bivalent fHBP vaccine to prevent Neisseria meningitidis serogroup $\mathrm{C}$ Disease. Hum Vaccin 2011:7(Suppl):68-74. 